\title{
PREFACE
}

The idea of writing a guide to Central American carnivores came to us after spending many years working as researchers and conservationists in the Neotropics. Together we have spent over 13 years involved in several aspects of tropical biology, research, education, and conservation, mostly in Costa Rica and Nicaragua. In recent years, we have come to realize that the status of many species of tropical carnivores is critical. Some of the largest and most recognizable species, like the jaguar and the puma, have suffered tremendous losses to their habitats. Some of these losses have become the main reason why these species are doomed to disappear entirely from many parts of their ranges. While conservation efforts in Central America are progressing steadily throughout the region, for some of these species, the progress simply is not happening fast enough.

In this book we make some sobering observations and claims as to the future of several species of carnivores in the region. These observations are based on the present knowledge of the biological needs of the species and the immediate prospects for habitat conservation and restoration. While the situation is not terribly urgent for many species of Neotropical carnivores, for several of them the next Io or I 5 years will decide their future presence and sustainability or their disappearance from the region. 
Regional efforts, such as the Central American Biological Corridor, or Paseo Pantera, are initiatives in the right direction, although the difficulties in establishing multipurpose biological corridors through an assortment of countries, economies, and cultures are numerous and complex. Conservationists tend to become involved in politics, sociology, and economics, often to the point of losing sight of what is actually best for the species and habitats they are trying to preserve. If we really want to make progress in the conservation of carnivores and their habitats, biological information about the species should be made widely available to all segments of society, not just to biologists. Politicians, economists, sociologists, and conservationists need to speak the same language. Conservation in the American tropics involves much more than choosing where parks, preserves, and biological corridors should be established. It also requires striking a balance among the specific needs of a dozen countries (some outside Central America, but with strong influences in the region) and taking into account their economic and social pressures, as well as incorporating the needs of future generations in the decision-making process.

We hope that the information in this guide, and in similar guides to come, helps bring relevant biological information to the discussion and negotiation table. Rarely is the biologist the one who decides where a conservation area will be located, nor is it the biologist who writes the laws or attempts to enforce them. This guide, then, is written with the nonbiologist in mind. Most technical terms are either explained in the text when first used or included in the glossary.

\section{HOW TO USE THIS BOOK}

This guide is divided into three parts. The introductory chapter deals with general characteristics of Central American carnivores, including their evolution, taxonomy and classification, distribution, habits, and other topics, such as vegetation types and tracking. Following the introduction, each family of Central American carnivore is presented, beginning with an introduction for each family and followed by a description of each species. We include with each description a map of species distribution where the reader can find the areas most likely to have representative populations of the species. These distribution maps differ from those commonly found in most field guides in that, in addition to showing the historic range for the species, we include the major patches of suitable habitats where the species actually can be found at present. In general terms, these patches correspond to protected areas, both private and government-supported (for example, national parks), or unprotected areas with 
various types of natural vegetation cover. We make no attempt to qualify these protected areas as suitable or unsuitable for specific species; as we will show in the species accounts, there is much to be learned about the habitat requirements, territories, and home ranges for most species, and this kind of information-which only long-term research can provide-is essential for managing these areas and maintaining viable populations of each species. At the end of each species description there is a conservation status summary of the species intended for quick reference. Finally, a chapter on carnivore conservation explores some of the major carnivore conservation topics and presents other pressing issues relating to the future of carnivores in Central America.

We have strived to include the latest research findings and taxonomic changes in this guide. However, carnivore taxonomy and systematics, as well as ecological research, is a very active and continuously changing field, and not all of the players are in agreement with the findings of their colleagues. We have included in the species accounts only that information which has been published in peer-reviewed scientific journals, books from recognized and reviewed publishing houses, and reports and publications from agencies involved in carnivore conservation, such as the International Union for the Conservation of Nature (IUCN), the World Wildlife Fund (WWF), and others. Our firsthand accounts of encounters with individuals of most species are intended to give insights into the lives and habits of these creatures, the places where they live, and some of the risks to which they are exposed in Central America. If these descriptions succeed in raising readers' interest in knowing a little more about these species, our goal will have been fulfilled. 
THIS PAGE INTENTIONALLY LEFT BLANK 